\title{
Anomalous phase transformation behavior and hyperfine ferromagnetism inCo; results from perturbed angular correlation spectroscopy corroborated byab-initio calculations
}

C. C. Dey ( $\nabla$ chandicharan.dey@saha.ac.in )

Saha Institute of Nuclear Physics

\section{R. Sewak}

Saha Institute of Nuclear Physics

D. Toprek

Vinca Institute of Nuclear Sciences, National Institute of the Republic of Serbia, University of Belgrade, P. O. Box 522, 11001, Belgrade, Serbia

\section{Research Article}

Keywords:

Posted Date: February 17th, 2022

DOI: https://doi.org/10.21203/rs.3.rs-1279244/v1

License: (c) (1) This work is licensed under a Creative Commons Attribution 4.0 International License.

Read Full License 


\title{
Anomalous phase transformation behavior and hyperfine ferromagnetism in Co; results from perturbed angular correlation spectroscopy corroborated by ab-initio calculations
}

\author{
R. Sewak ${ }^{1,2}$, C. C. Dey ${ }^{1,2, *}$, and D. Toprek ${ }^{3}$ \\ ${ }^{1}$ Saha Institute of Nuclear Physics, 1/AF Bidhannagar, Kolkata-700064, India \\ ${ }^{2}$ Homi Bhabha National Institute, Anushaktinagar, Mumbai-400094, India \\ ${ }^{3}$ Vinca Institute of Nuclear Sciences, National Institute of the Republic of Serbia, University of Belgrade, P. O. Box \\ 522, 11001, Belgrade, Serbia \\ *chandicharan.dey@saha.ac.in
}

\begin{abstract}
Temperature dependent phase transformation behavior in Co from hcp to fcc has been found to be contradictory to that reported earlier. It is found that hcp phase stabilizes at both low and high temperature $(\sim 873 \mathrm{~K})$ while fcc phase is stabilized at $\sim 500$ K. At $298 \mathrm{~K}$, hcp Co has been found to be predominant ( 70\%) where hcp magnetic phase is $\sim 60 \%$. At $973 \mathrm{~K}$, hcp phase is again predominant $(\sim 73 \%)$, but it is mainly the non-magnetic phase $(\sim 67 \%)$. Contrary to present results, it was found earlier that fcc phase was stabilized at high temperature and hcp to fcc transformation occured at $\sim 700 \mathrm{~K}$. Theoretical models show that non-magnetic fcc phase is more stable than non-magnetic hcp phase and presence of magnetism destabilizes the fcc phase at low temperature. Present results from perturbed angular correlation measurements, therefore, requires a new theoretical interpretation for Co phase transformation. From present measurements, hyperfine magnetic fields in Co at room temperature for the hcp and fcc phases have been found to be 18.7(6) and 12.8(3) T, much lower than earlier reported results. The hyperfine magnetic fields at ${ }^{181} \mathrm{Ta}$ impurity atom have been calculated by density functional theory (DFT) employing the full potential (linearized) augmented plane wave method (FP-LAPW). Present calculated results for both hcp and fcc phases corroborate our experimental results.
\end{abstract}

\section{Introduction}

The elemental Co is known to have ferromagnetism at room temperature. It has two crystal structures of hexagonal close-packed (hcp) and face centred cubic (fcc). At low temperature, Co crystallizes in a hcp lattice with a c/a ratio $=1.62$ at $298 \mathrm{~K}$, very close to the ideal value $(c / a=1.633)$ for closet packing. It was found ${ }^{1}$ that Co undergoes a phase transition from hcp to fcc just below $700 \mathrm{~K}$. From hyperfine interaction studies of perturbed angular correlation (PAC) using ${ }^{111}$ In probe, Lindgren et al. ${ }^{2}$ reported that Co has a pure hcp phase at $\leq 604 \mathrm{~K}$ while it has a pure fcc phase at $771 \mathrm{~K}$. In the intermediate $648 \mathrm{~K}$, both hcp and fcc phases were found to co-exist ${ }^{2}$. In a subsequent report by Bedi et al. ${ }^{3}$ using the same hyperfine interaction method but with a different ${ }^{181} \mathrm{Hf}$ probe, however, it was shown that the hcp phase was present up to $700 \mathrm{~K}$ and due to the sluggish nature of this phase transition, both hcp and fcc phases were found to be present at room temperature and above. From previous PAC measurements using ${ }^{181} \mathrm{Hf}$ probe ${ }^{3}$, the hcp phase was found to be $\sim 66 \%$ at room temperature along with a significant fraction $(\sim 5-10 \%)$ for the fcc phase. The presence of fcc fraction at room temperature was confirmed also from X-ray diffraction pattern.

The magnetism of Co was found for both these phases ${ }^{2,3}$. At room temperature, the magnetic field strength for the hcp phase was found to be approximately $10 \%$ higher than the fcc phase ${ }^{3}$. It was reported that both the structures of hep and fcc have room temperature ferromagnetism at the probe Ta impurity site ${ }^{3}$. From earlier reports ${ }^{2,3}$, a higher value of hyperfine magnetic field for the hcp phase was found compared to the fcc phase. From the previous report by Lindgren et al. ${ }^{2}$ no fcc phase fraction was found at room temperature while a definite value of hyperfine magnetic field for the fcc phase was reported by Bedi et al. ${ }^{3}$. It was shown earlier ${ }^{4-7}$ that magnetism plays an important role in the phase stability of $3 \mathrm{~d}$ transition metals. From calculations by density functional theory (DFT) ${ }^{8}$, it was found that if Co was not magnetic, it would choose the fcc phase as its ground state. But, presence of magnetism lowers the total energy of the hcp phase. 
The temperature induced structural phase transition in Co from hcp to fcc is not properly understood. It was reported ${ }^{9}$ that absence of magnetism at high temperature induced this phase transition. It is generally considered that the hcp ground state in Co is stabilised in presence of magnetism. At room temperature, the cobalt phase is mainly hcp and the magnetism in Co is also maximum at room temperature and below it. The reduction of magnetism at high temperature destabilizes the hcp phase $^{7,9,10}$. Uhl and Kübler ${ }^{7,11}$, from theoretical calculations, showed that spin fluctuation and reduced magnetism at high temperatures lower the free energy of the fcc phase and triggers the hcp to fcc phase transition. From their calculations, they reported a transition temperature of $590 \mathrm{~K}$ by considering the magnetic effects only. But, it is recently shown ${ }^{8}$ that reduced

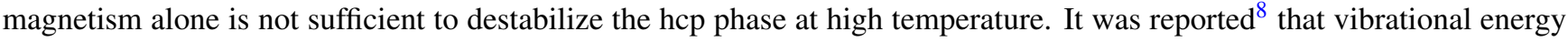
arising due to vibration of ionic lattice is the main driving force for the hcp to fcc phase transition and besides this, other free energy terms like magnetic, electronic and volume effects must be included to understand this phase transition properly. By including all these contributions to free energy, they reported a transition temperature of $825 \mathrm{~K}$. Therefore, to understand the main driving force that causes the hcp to fcc phase transition in $\mathrm{Co}$, it is very important to determine the temperature induced phase transformation behavior. Considering discrepancies of results in the previous reports ${ }^{2,3}$, temperature dependent PAC measurements in Co using ${ }^{181} \mathrm{Hf}$ probe have been re-investigated in the present report.

The hcp crystalline phase has a non-cubic symmetry and the charge distribution surrounding the probe generates an electric field gradient (EFG) at the probe impurity site. Therefore, a combined magnetic and electric quadrupole hyperfine interaction is expected in hcp Co at room temperature. Above Curie temperature, when there is no ferromagnetism, only a pure quadrupole interaction is expected. For the fcc phase, however, no hyperfine electric quadrupole interaction is expected above the Curie temperature and there will be no perturbation in the PAC spectrum.

The PAC is a useful nuclear technique for the studies of structural and magnetic phase transitions in materials ${ }^{12-14}$ where a suitable radioactive nucleus is inserted as a probe within the material. For present studies, we have used ${ }^{181} \mathrm{Hf}$ probe decaying to ${ }^{181} \mathrm{Ta}$ through $\beta^{-}$. The daughter nucleus has a strong $133-482 \mathrm{keV} \gamma-\gamma$ cascade passing through the $482 \mathrm{keV}$ level $\left(I=5 / 2^{+}\right.$, $\mathrm{T}_{1 / 2}=10.5 \mathrm{~ns}$ ) having high values of electromagnetic moments ${ }^{15}$. In a non-cubic environment of the probe, the electric quadrupole moment of the probe interacts with the surrounding electric field gradient and in a magnetic material, the magnetic field interacts with the surrounding magnetic field. Due to these interactions, the selected angular correlation (133-482 keV cascade) is perturbed.

The perturbation function corresponding to static pure electric quadrupole interaction, for a polycrystalline sample and for $I=5 / 2$ intermediate level is given by ${ }^{13,14}$

$$
G_{22}(t)=S_{20}(\eta)+\sum_{n=1}^{3} S_{2 n}(\eta) \cos \left(\omega_{n} t\right) \exp \left(-\delta \omega_{n} t\right)
$$

The coefficients $\mathrm{S}_{2 n}{ }^{16}$ depend upon orientation of the electric field gradient (EFG) tensor and are different for single and polycrystalline samples. The parameter $\delta$ represents the Lorentzian shape damping of the quadrupole frequency distribution due to imperfections in crystal lattice structure (defects, impurities, etc.). The electric field gradient in the principle axis system can be described by two parameters. i) The quadrupole frequency $\left(\omega_{Q}\right)$ and asymmetry parameter $\eta^{16}$.

If the probe nuclei experience a pure magnetic field (in a cubic crystalline environment), the corresponding magnetic perturbation can be described by

$$
G_{22}(t)=1 / 5\left[1+2 \cos \left(\omega_{L} t\right)+2 \cos \left(2 \omega_{L} t\right)\right]
$$

Here, $\omega_{L}$ is the Larmor precession frequency (proportional to the hyperfine magnetic field) and is given by $\omega_{L}=g \mu_{N} B_{h f} / \hbar$. From the measured PAC spectrum $\left(G_{22}(t)\right.$ vs $\left.t\right)$, values of $\omega_{L}$ can be determined. The $g$ factor of the probe nuclear level is related to the nuclear magnetic moment by $\mu=g I, \mu$ is nuclear magnetic moment of the intermediate level and $B_{h f}$ is the effective hyperfine magnetic field experienced by probe nuclei in the sample and $I$ is spin angular momentum of the intermediate level.

In a non-cubic site environment, the probe nucleus experiences a combined magnetic dipole and electric quadrupole hyperfine interaction. The perturbation function due to this combined interaction depends on five interaction parameters and on the nuclear spin $I^{17-19}$. In this case, the interaction parameters are the magnetic frequency $\omega_{L}$, the quadrupole frequency $\omega_{Q}$, the asymmetry parameter $\eta$, the Euler angles $\theta$ and $\phi$ which describe the relative orientation of the magnetic hyperfine field and the EFG tensor.

\section{Experimental details}

For present PAC measurements, a four detector time differential perturbed angular correlation (TDPAC) set up with two $\mathrm{LaBr}_{3}(\mathrm{Ce})\left(38 \times 25.4 \mathrm{~mm}^{2}\right)$ detectors and two $\mathrm{BaF}_{2}\left(50.8 \times 50.8 \mathrm{~mm}^{2}\right)$ detectors have been used. Details about data collection 
Table 1. PAC results for Co at three selected temperatures.

\begin{tabular}{|c|c|c|c|c|c|c|c|c|}
\hline \multirow{2}{*}{$\begin{array}{l}\text { Temp. } \\
\text { (K) }\end{array}$} & \multirow{2}{*}{ Comp. } & \multicolumn{4}{|c|}{ Quadrupole interaction } & \multicolumn{3}{|c|}{ Magnetic dipole interaction } \\
\hline & & $\begin{array}{c}\omega_{Q} \\
(\mathrm{Mrad} / \mathrm{s})\end{array}$ & $\eta$ & $\begin{array}{c}\delta \\
(\%)\end{array}$ & $\begin{array}{c}f \\
(\%)\end{array}$ & $\begin{array}{c}\omega_{L} \\
(\mathrm{Mrad} / \mathrm{s})\end{array}$ & $\begin{array}{c}\delta \\
(\%)\end{array}$ & $\begin{array}{c}f \\
(\%)\end{array}$ \\
\hline \multirow[t]{4}{*}{298} & 1 & $58.9(4)$ & $0.38(2)$ & 0 & $9(1)$ & & & \\
\hline & 2 & $16(1)$ & 0 & 0 & $9(1)$ & & & \\
\hline & 3 & & & & & $1154(25)$ & $27(7)$ & $65(3)$ \\
\hline & 4 & & & & & $823(7)$ & $5(2)$ & $17(1)$ \\
\hline \multirow[t]{4}{*}{$298^{\dagger}$} & 1 & $59.5(3)$ & $0.39(2)$ & 0 & $10(1)$ & & & \\
\hline & 2 & $13.8(6)$ & 0 & 0 & $9(1)$ & & & \\
\hline & 3 & & & & & $1187(24)$ & $22(5)$ & $60(3)$ \\
\hline & 4 & & & & & $810(7)$ & $9(2)$ & 21(1) \\
\hline \multirow[t]{4}{*}{873} & 1 & $67.5(2)$ & $0.16(2)$ & $2.5(6)$ & $64(4)$ & & & \\
\hline & 2 & $17(1)$ & 0 & 0 & $11(1)$ & & & \\
\hline & 3 & & & & & $935(11)$ & 0 & $5(1)$ \\
\hline & 4 & & & & & $674(3)$ & 0 & $20(3)$ \\
\hline \multirow[t]{4}{*}{973} & 1 & $67.1(2)$ & $0.16(2)$ & $3.0(6)$ & $67(4)$ & & & \\
\hline & 2 & $16(1)$ & 0 & 0 & $8(1)$ & & & \\
\hline & 3 & & & & & $872(8)$ & 0 & $6(1)$ \\
\hline & 4 & & & & & $676(2)$ & 0 & $19(1)$ \\
\hline
\end{tabular}

$\dagger$ after annealing the sample.

Components 1 and 3 assigned to hcp while 2 and 4 assigned to fcc phase

and generation of $\mathrm{A}_{22} \mathrm{G}_{22}(\mathrm{t})$ from the four coincidence spectra at $180^{\circ}$ and $90^{\circ}$ can be found in our earlier report ${ }^{16}$. The program WINFIT ${ }^{20}$ was used to fit the PAC spectra. In the fitting, we assume there is no interference on the magnetic hyperfine frequency by the electric quadrupole frequency. This is a special situation and can be justified by considering the fact that the Larmor precession frequency is much larger than the electric quadrupole frequency i.e. $\omega_{L} \gg \omega_{Q}$. In the analysis, we therefore consider the electric and magnetic frequency components are independent to each other and these different components are simply combined to get the total PAC spectrum.

The sample for PAC measurement was prepared by arc melting high purity $(99.995 \%)$ cobalt wire with a tiny piece of active ${ }^{181} \mathrm{Hf}$ wire in argon atmosphere. The probe concentration in the sample $(\sim 0.3$ at. $\%)$ was too small to affect the bulk sample properties. The active ${ }^{181} \mathrm{Hf}$ was produced from natural $\mathrm{Hf}$ by thermal neutron capture at Dhruba reactor (Mumbai), India.

\section{Results and discussion}

From PAC spectrum at room temperature four frequency components have been found. Results for the four components in as prepared sample and also in annealed sample are shown in table 1 . The sample was annealed at $773 \mathrm{~K}$ for one day. It is found that the PAC spectra and the results before and after annealing do not change appreciably. The PAC spectrum found after annealing the sample is shown in figure 1. In annealed sample, the major component $(\sim 60 \%)$ gives $\omega_{L}=1187(24) \mathrm{Mrad} / \mathrm{s}$, with a large value of frequency distribution width $(\delta \sim 22 \%)$. This has been attributed to hyperfine magnetic field corresponding to hcp Co. It is known that Co has a hcp crystal structure at room temperature and a ferromagnetism is expected to be stronger at low temperature. The magnetic frequency component with a lower value of $\omega_{L}$ has been attributed to fcc Co. It is already known $^{2,3}$ that hyperfine magnetic field in fcc Co is lower than the hcp Co. The two quadrupole frequency components found have been assigned also to hcp and fcc crystal structures of Co. For annealed sample, the quadrupole frequency component with values of $\omega_{Q}=59.5(3) \mathrm{Mrad} / \mathrm{s}, \eta=0.39(2), \delta=0$ has been attributed to hcp Co and the lower frequency component (table 1) with values of $\omega_{Q}=13.8(6) \mathrm{Mrad} / \mathrm{s}, \eta=0, \delta=0$ has been attributed to fcc Co. A pure fcc Co should not produce any EFG. But, in this case, we are measuring the EFG at the probe impurity site where the local site symmetry is broken in presence 

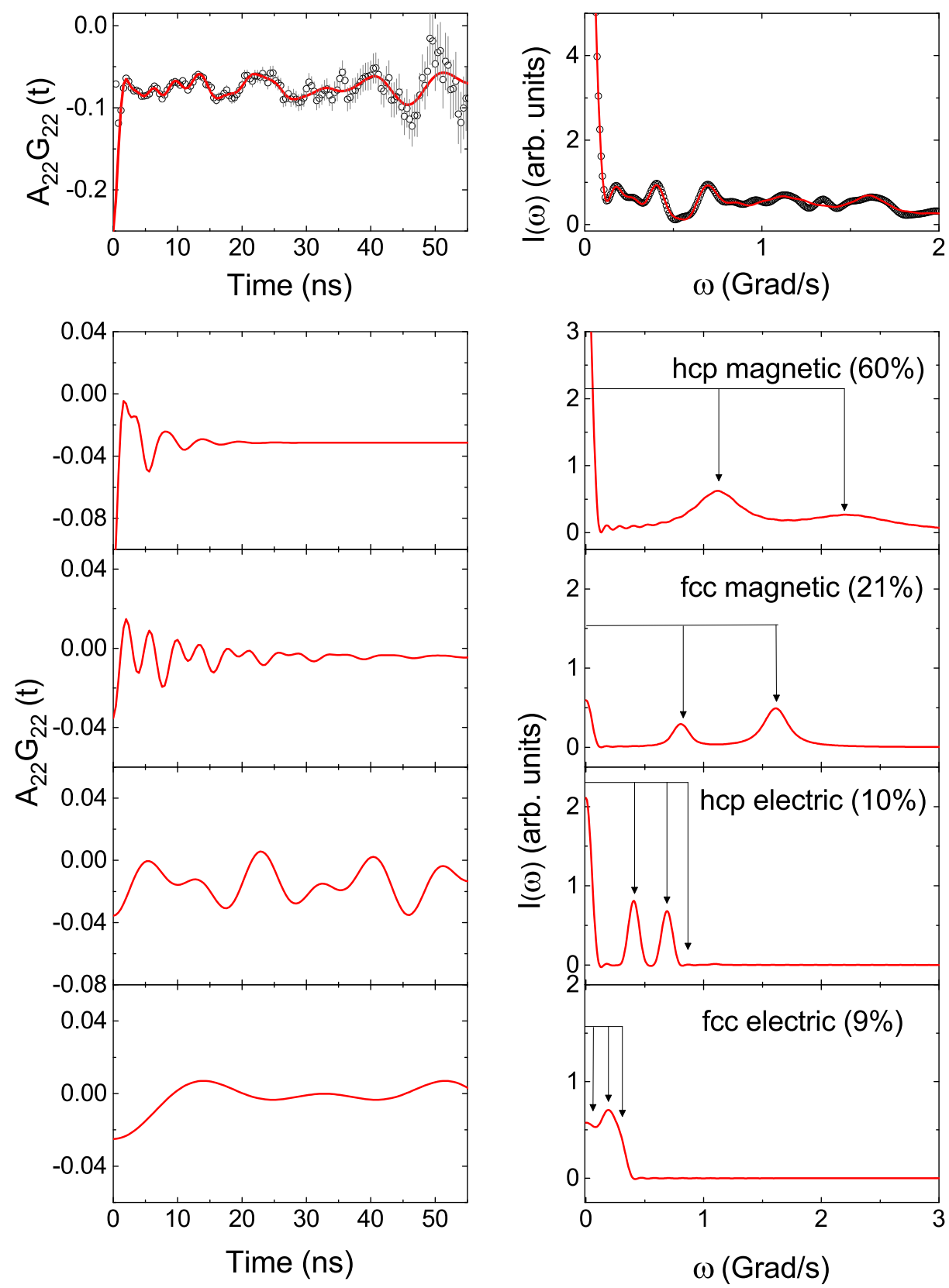

Figure 1. Total PAC spectrum at $298 \mathrm{~K}$ in annealed sample (top) and its decomposed spectra (bottom); Left panel shows time spectra and right panel shows corresponding Fourier transforms 
of impurity and a non-cubic symmetry is generating a finite EFG. The decomposed four spectra corresponding to these four components are shown in figure 1 . The hcp magnetic component is clearly found to be the predominant component. But, the hcp electric quadrupole component and the two fcc components (magnetic and electric quadrupole) are clearly observed at room temperature. The total fcc phase at room temperature has been found to be quite large $(\sim 28 \%)$. The results in pre-annealed sample are also found to be similar (table 1).
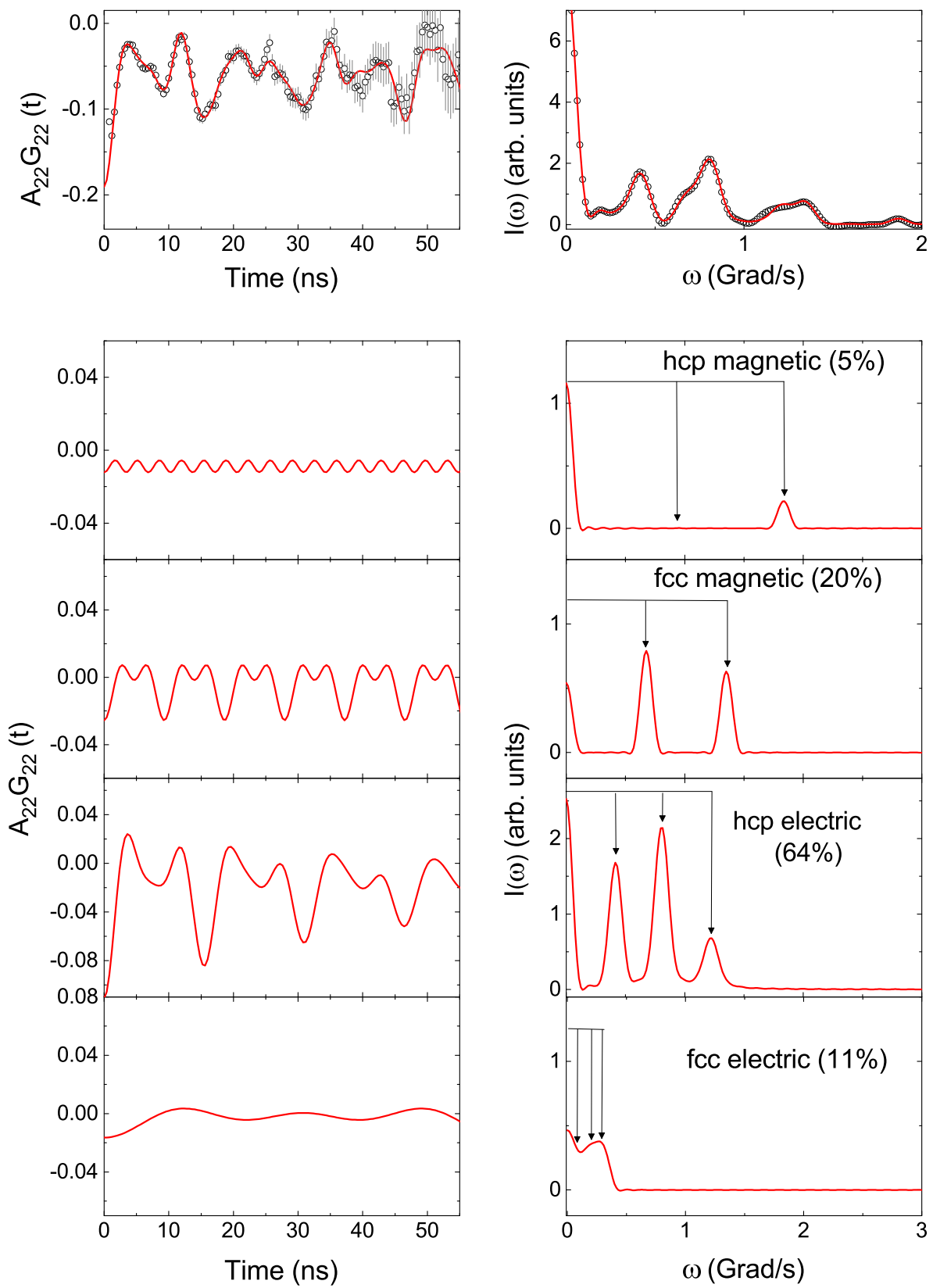

Figure 2. Total PAC spectrum at $873 \mathrm{~K}$ (top) and its decomposed spectra (bottom); Left panel shows time spectra and right panel shows corresponding Fourier transforms

The fcc fraction in Co was found at room temperature from previous report ${ }^{3}$ also. From X-ray diffraction studies, it was reported to be present with a small fraction (5-20\%) at room temperature. From the other report ${ }^{2}$, however, no fcc phase was found at room temperature and the present results contradict with the results reported in reference ${ }^{2}$. The hyperfine magnetic field strengths for the hep and fcc phases at room temperature have been found to be 18.7(6) T and 12.8(3) T, respectively for the present measured values of $\omega_{L}$ in annealed sample (considering a value of $g=1.31(3)^{15}$ for the $482 \mathrm{keV}$ level). While 


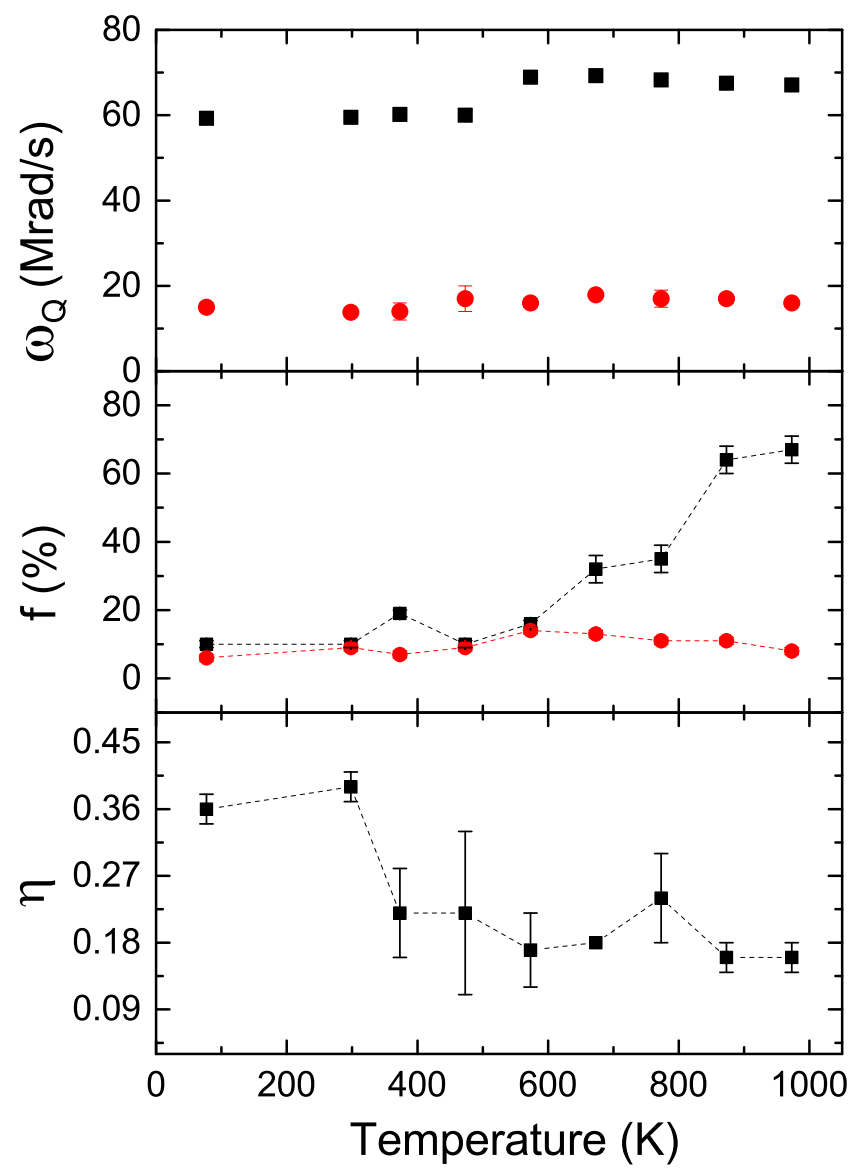

Figure 3. Variations of $\omega_{Q}$, site fraction (f) and $\eta$ with temperature for the hcp (filled square) and fcc (filled circle) phases.

the magnetic field strength for the hcp phase is higher than the fcc phase as reported earlier ${ }^{2,3}$, the present measured value of hyperfine magnetic field for the hcp phase is only $\sim 46 \%$ to that reported by Bedi et al. ${ }^{3}$ and for the fcc phase, it is only $\sim 35 \%$. The corresponding hyperfine magnetic fields found from previous measurement ${ }^{3}$ were 40.7 and $36.3 \mathrm{~T}$ for hcp and fcc phases, respectively.

To determine the variations of hyperfine magnetic fields and electric field gradients with temperature, we have done measurements at a lower temperature of $77 \mathrm{~K}$ and at higher temperatures. At $77 \mathrm{~K}$ also, these four components have been observed and the results do not change much compared to the results found at room temperature. Interestingly, at high temperature $(\geq 873 \mathrm{~K})$, a drastic change in the PAC spectrum has been found (figure 2). At $873 \mathrm{~K}$, all four components have been found but, their site fractions are completely changed (table 1). At this temperature, the hcp quadrupole frequency component is predominant $(\sim 64 \%)$ while the hcp magnetic component fraction has been found as a negligible fraction $(\sim 5 \%)$. The results remain almost same at $973 \mathrm{~K}$. At temperatures $\sim 500 \mathrm{~K}$, on the other hand, the hcp magnetic fraction is much lower than the fcc magnetic fraction (figure 4).

In the temperature range 77-973 K, variations of $\omega_{Q}, \eta$ and site fractions with temperature for the hcp and fcc phases of Co are shown in figure 3. For both these fractions, $\delta$ were found to be zero up to $773 \mathrm{~K}$. At 873 and $973 \mathrm{~K}$, strength of quadrupole interaction increases with small values of frequency distribution width. Variations of $\omega_{L}, \delta$ and corresponding site fractions with temperature for the two magnetic phases are shown in figure 4. It is found that at room temperature, the hcp magnetic fraction is predominant but at $973 \mathrm{~K}$, the hcp quadrupole fraction is predominant. Values of $\omega_{Q}$ for both hcp and fcc components do not change in the temperature range 77-500 K. However, for the hcp component, it shows a clear discontinuity at $\sim 500 \mathrm{~K}$ and at this temperature the value of $\omega_{Q}$ increases by approximately $10 \%$ compared to the room temperature value. In the temperature range 500-973 K, the enhanced values of $\omega_{Q}$ remain unchanged. This change in quadrupole frequency can be understood by the fact that from around $500 \mathrm{~K}$, the total electric quadrupole fraction increases rapidly at the expense of magnetic fraction (figure 5). Probably, at lower temperatures $(\mathrm{T} \leq 500 \mathrm{~K})$, the quadrupole frequencies have been modified by the stronger magnetic components. At temperatures $\mathrm{T}>500 \mathrm{~K}$, the magnetic component fraction is not sufficiently strong to 


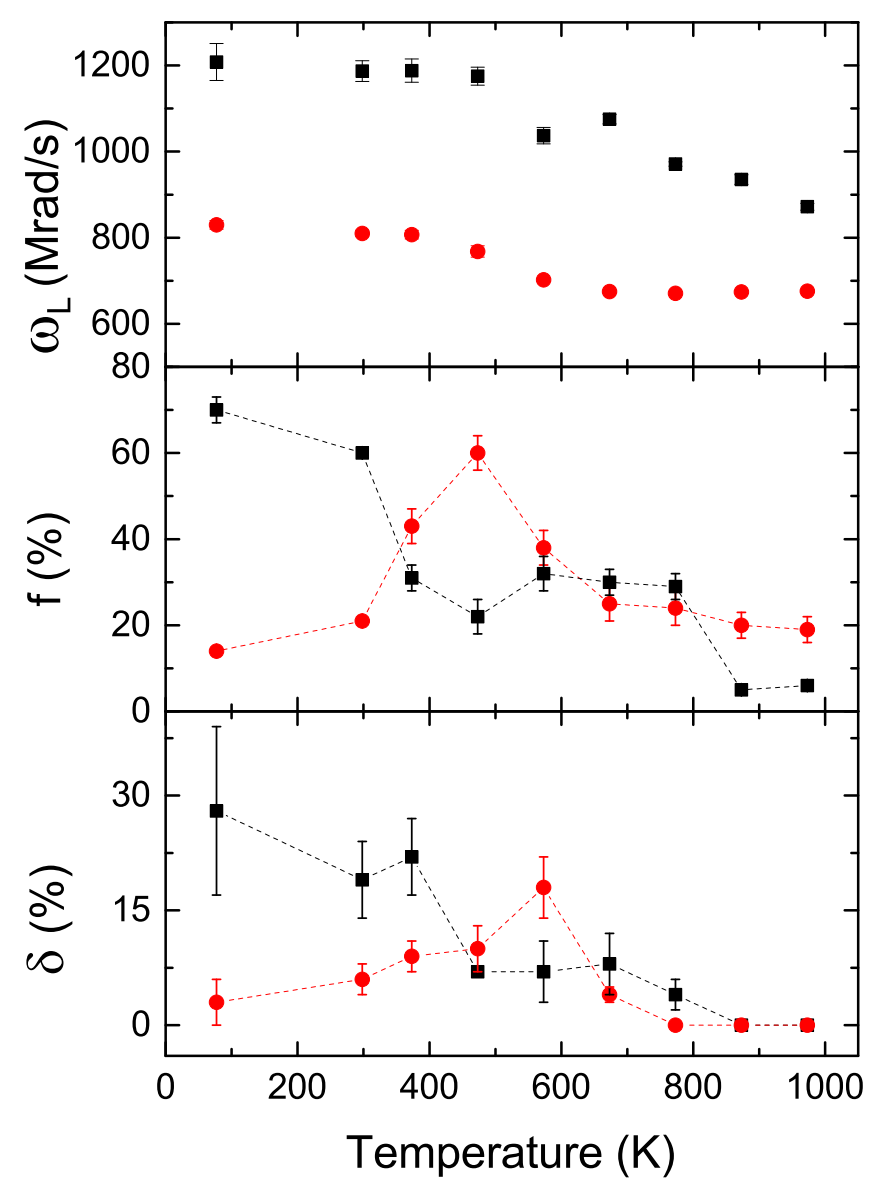

Figure 4. Variations of $\omega_{L}$ along with their site fraction (f) and $\delta$ with temperature for the hcp (filled square) and fcc (filled circle) phases.

modify the quadrupole frequency of the hcp component. For the fcc component, this is not clearly observed because of lower values of $\omega_{Q}$, although it shows an increasing tendency in the higher temperature region (500-973 K).

Values of $\omega_{L}$ are found to clearly decrease with temperature as expected. For the hcp phase, $\omega_{L}$ decreases with temperature in the high temperature region (above $400 \mathrm{~K}$ ). For the fcc phase, however, $\omega_{L}$ remains almost same in the temperature range $600-973 \mathrm{~K}$. This is quite unexpected and the reason is not clearly understood. A vanishingly small site fraction for the hcp magnetic field at $973 \mathrm{~K}$ indicates that the Curie temperature for this phase is $\sim 975 \mathrm{~K}$ and it agrees well with the value of $\mathrm{T}_{c}$ reported earlier ${ }^{3}$. For the fcc phase, however, a relatively large site fraction for the fcc magnetic component at $973(\sim 19 \%)$ has been found suggesting a higher Curie temperature for the fcc phase as found earlier.

Temperature dependent results of $\omega_{L}$ for hcp and fcc phases have been tried to fit with the power law given by

$$
\omega_{L}(T)=\omega_{L}(0)\left[1-\frac{T}{T_{c}}\right]^{\beta}
$$

It is found that for fcc phase, the data are not fitted well in the higher temperature region (figure 6). It seems that the power law variation of $\omega_{L}$ with temperature can not describe the results well in the whole temperature region. In the intermetallic $\mathrm{ZrFe} 2$ $\mathrm{also}^{21}$, this type of relation was not found to be valid in the whole temperature region. However, if we consider that this power law variation is an approximate relation, results for the hcp phase in Co gives a higher value of $T_{c}(\sim 1568 \mathrm{~K})$ than the value of $T_{c}$ for the fcc phase $(\sim 1053 \mathrm{~K})$. From previous measurement ${ }^{3}$, the Curie temperature for fcc phase, on the other hand, was reported to be higher than the hcp phase. From present results also, a higher value of $T_{c}$ for fcc phase than hcp phase can be estimated, considering higher fractions of fcc magnetic component than the corresponding hcp magnetic component at 873 and $973 \mathrm{~K}$ and almost no change of $\omega_{L}$ for fcc phase in the temperature region 773-973 K. Actually, by fitting with power law, the results of $\omega_{L}$ for fcc phase in the higher temperature region (773-973 K) were not considered and could be the reason for this discrepancy. 


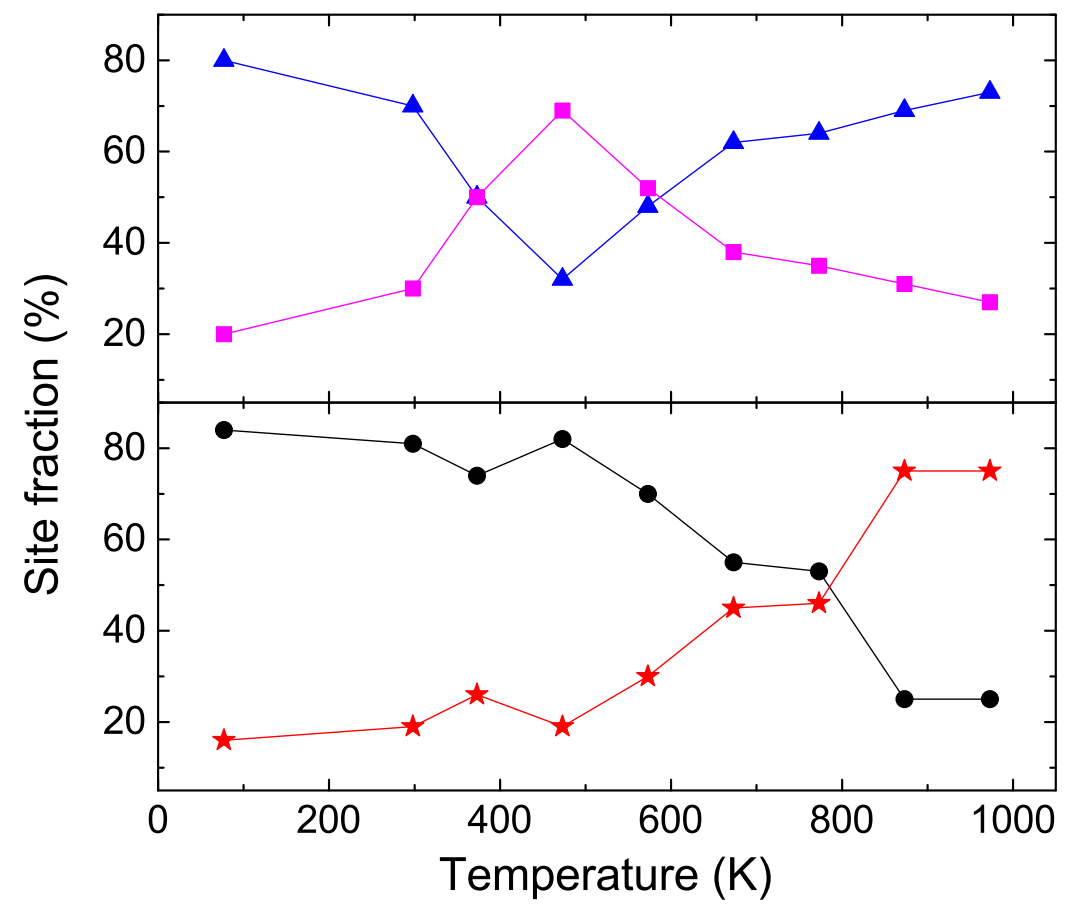

Figure 5. Top: Variations of site fractions for total hcp (triangle) and total fcc (square) phases with temperature. Bottom: Variations of site fractions for total magnetic (circle) and total non-magnetic (star) components with temperature.

Variations of total site fractions (magnetic and non-magnetic component together) with temperature for the hcp and fcc phases are shown in figure 5. It is found that the total hep fraction first decreases with temperature and shows a minimum at $\sim 500 \mathrm{~K}$. Above $500 \mathrm{~K}$, the hcp fraction increases again with temperature and found to be maximum at around $973 \mathrm{~K}$. On the other hand, the total fcc site fraction is $\sim 30 \%$ at room temperature. Up to $500 \mathrm{~K}$, this phase increases with temperature and then decreases again. The total fcc fraction has been found to be maximum ( $\sim 70 \%)$ at $473 \mathrm{~K}$. At $\sim 873 \mathrm{~K}$ and above, the fcc phase again shows as a minor fraction. The figure shows that at low temperature and also at high temperature (above $873 \mathrm{~K}$ ), the hcp is found as a predominant phase while in the temperature range $373-573 \mathrm{~K}$, the fcc phase is found to be more stable than the hcp phase. After measurement at $973 \mathrm{~K}$, a remeasurement at $298 \mathrm{~K}$ was carried out where the results are found to be reversible. Variations of total magnetic (hcp and fcc together) and non-magnetic fractions with temperature are also shown in figure 5. It is found that up to $500 \mathrm{~K}$, the total magnetism does not change much. After $\sim 500 \mathrm{~K}$, the magnetic component decreases and the non-magnetic component increases with temperature. So, in the temperature region $(77-500 \mathrm{~K})$ where the total magnetism remains almost unchanged, the phase fraction changes from hcp to fcc. Also, when the magnetism is low at high temperature $(\geq 873 \mathrm{~K})$, the hcp phase fraction again shows its maximum value. From present results it appears that magnetism has no role in the phase stabilization.

Present results contradict with the results reported in reference ${ }^{2}$, where a pure fcc magnetic phase was found at $771 \mathrm{~K}$ and also contradict with the behavior of phase fractions with temperature as reported by Bedi et al. ${ }^{3}$. The reason for discrepancies could be attributed to experimental inaccuracy and also to wrong analysis of data in previous reports. In fact, determination of correct results from the much complicated PAC spectra where there are four frequency components (two magnetic and two quadrupole) and finding their variations with temperature are really challenging. Unless the data analysis are correctly done, results can be found which are far from reality. In present case, much efforts have been given to determine the results correctly. It can be mentioned here that from PAC measurements only, simultaneous variations of magnetic and non-magnetic components with temperature for the two phases can be determined and, therefore results from PAC measurements, can be considered as more accurate than other experimental techniques. The results reported by Bedi et al. ${ }^{3}$ are difficult to understand from following considerations. Starting a pure $\mathrm{Co}$ and using ${ }^{181} \mathrm{Hf}$ probe, these authors ${ }^{3}$ reported a pure quadrupole interaction due to production of intermetallic compound $\mathrm{Hf}_{2} \mathrm{Co}_{7}$ at $700 \mathrm{~K}$ and a different compound of $\mathrm{Hf}_{6} \mathrm{Co}_{23}$ at $900 \mathrm{~K}$. But, productions of these intermetallic compounds at high temperature seems to be improbable. This is because, i) in their samples, the Hf concentrations were $\sim 0.2$ at $\%$ and at this very small $\mathrm{Hf}$ concentration, production of any intermetallic compound with Co is unlikely. ii) If any intermetallic compound is locally formed with $\mathrm{Co}$, it should not be the pure single component, rather it should be a minor component, major component should be due to Co. iii) If it is assumed that the $\mathrm{Hf}_{2} \mathrm{Co}_{7}$ is formed at 700 


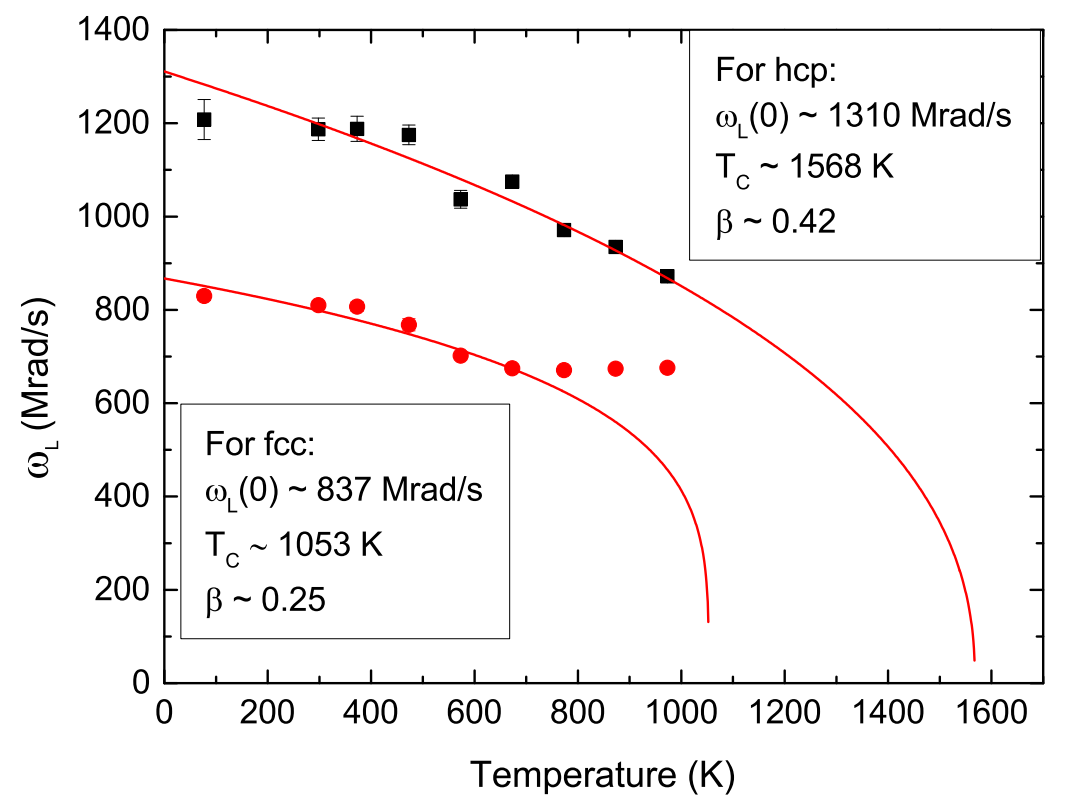

Figure 6. Temperature dependent behaviors of $\omega_{L}$ fitted with Eqn. 3 for hcp (filled square) and fcc (filled circle) phases in Co.

$\mathrm{K}$, it is not understood why it transforms to another compound $\mathrm{Hf}_{6} \mathrm{Co}_{23}$ at $900 \mathrm{~K}$ and then again to elemental Co at $1300 \mathrm{~K}$ (not consistent with the reported phase diagram also). iv) From our recent PAC measurements in $\mathrm{Hf}_{2} \mathrm{Co}_{7}$ and $\mathrm{Hf}_{6} \mathrm{Co}_{23}{ }^{22,23}$, completely different PAC spectra and results have been found than reported by Bedi et al. ${ }^{3}$. Due to these reasons, probably, the earlier measured hyperfine magnetic fields at Ta impurity (40.7 $\mathrm{T}$ and $36.3 \mathrm{~T}$ for hcp and fcc phases, respectively) are not reliable.

To confirm our experimental results, we have calculated the hyperfine electric field gradients and magnetic fields at the ${ }^{181} \mathrm{Ta}$ impurity site by density functional theory (DFT) employing the WIEN2K code in the framework of full potential (linearized) augmented plane wave method. The details about the calculation is given in the following section. Our calculated and experimental results are shown in table 2. The calculated results for the site Hf-Ta1 are shown in the table for comparing with our experimental results. It is found that for the hcp phase, the calculated result of $V_{z z}$ perfectly agree with our experimental value at room temperature although the calculated result of asymmetry parameter $(\eta \sim 0.7)$ is higher that the experimentally observed value $(\eta \sim 0.4)$. For the fcc phase also, the calculated result of $V_{z z}$ is in good agreement with our experimental value. In this case, a zero value of asymmetry parameter is found experimentally as well as from DFT calculation. For the hcp hyperfine magnetic field, the calculated result comes out to be $-25.75 \mathrm{~T}$ (at $0 \mathrm{~K}$ ) which is comparable to the value found experimentally at room temperature $18.7 \mathrm{~T}$. However, if we extrapolate our experimental results to $0 \mathrm{~K}$ (by power law fitting), a value of $B_{h f}(0)=20.9 \mathrm{~T}$ (for $\omega_{L}=1310 \mathrm{Mrad} / \mathrm{s}$ ) is obtained which is more closer to the calculated result. For the fcc phase also, the present calculated result at $0 \mathrm{~K}(-19.99 \mathrm{~T})$ is closer to the experimental result extrapolated to $0 \mathrm{~K}(13.3 \mathrm{~T}$ for $\left.\omega_{L}=837 \mathrm{Mrad} / \mathrm{s}\right)$. On the other hand, a value of hyperfine magnetic field for the hcp phase at $9 \mathrm{~K}\left(B_{h f}=-40.7 \mathrm{~T}\right)$ was reported by Bedi et al. ${ }^{3}$ which is far away from the present calculated result. For the fcc phase also, the hyperfine magnetic field reported by Bedi et al. ${ }^{3}$ is much higher $(-36.3 \mathrm{~T}$ at $9 \mathrm{~K})$ than the calculated result. The present DFT calculated results of EFG and magnetic fields for both hcp and fcc phases, on the other hand, confirm our experimental results.

\section{DFT Calculation}

In this paper, we have performed the first-principles density functional theory (DFT) to calculate electric field gradients in term of largest diagonal component $V_{z z}$ and the asymmetry parameters $\eta$ in the pure Co as well as at Ta probe positions in the hep and fec crystal structure of Co. The hyperfine magnetic fields $\left(B_{h f}\right)$ have also been calculated at the Ta probe positions and for no probe condition. The lattice parameters $a, b, c, \alpha, \beta$ and $\gamma$, and the fractional coordinates of crystallographic non-equivalent positions of hcp P63/mmc (space group number: 194) and fcc $F m \overline{3} m$ (space group number: 225) crystal structure of Co are presented in the table 3 .

At first, we have optimized the structural parameters. We have employed WIEN $2 \mathrm{k}^{24}$ code where the full potential (linearized) augmented plane waves method (FP-LAPW) is implemented. The muffin-tin radii for Co was 1.6 a.u. The spherical 
Table 2. Comparison of calculated and experimental results of hyperfine $V_{z z}$ and $B_{h f}$ for hcp and fcc phases in Co at ${ }^{181}$ Ta impurity site

\begin{tabular}{|c|c|c|c|c|c|c|}
\hline \multirow{2}{*}{ Phase } & \multicolumn{2}{|c|}{$V_{z z}\left(10^{21} \mathrm{~V} / \mathrm{m}^{2}\right)$} & \multicolumn{2}{|c|}{$\eta$} & \multicolumn{2}{|c|}{$B_{h f}(\mathrm{~T})$} \\
\hline & $\mathrm{Cal}^{\dagger}{ }^{\dagger}$ & Exp. & $\mathrm{Cal}^{\dagger}{ }^{\dagger}$ & Exp." & $\mathrm{Cal}^{\dagger}$ & Exp. ${ }^{\S}$ \\
\hline hcp & -6.64 & 6.67 & 0.71 & 0.39 & -25.75 & 20.9 \\
\hline fcc & 0.9543 & 1.55 & 0.00 & 0 & -19.99 & 13.3 \\
\hline
\end{tabular}

$\dagger$ at $0 \mathrm{~K}$

$\$$ for annealed sample at $298 \mathrm{~K}$

$\S$ for annealed sample extrapolated to $0 \mathrm{~K}$

Table 3. The lattice parameters $\mathrm{a}, \mathrm{b}$ and c (given in $\AA$ ) and the fractional coordinates of crystallographic non-equivalent positions of hcp $P 63 / m m c$ (space group number: 194) and fcc $F m \overline{3} m$ (space group number: 225) crystal structure of Co getting by WIEN2k code ${ }^{24}$ (second and third columns) and these parameters taken from other research papers (fourth and fifth columns)

\begin{tabular}{cccccc}
\hline \hline \multirow{2}{*}{$\begin{array}{c}\text { Crystal } \\
\text { par. }\end{array}$} & \multicolumn{2}{c}{ WIEN2K calculation } & & \multicolumn{2}{c}{ Previous results $^{25,26}$} \\
\cline { 2 - 3 } \cline { 5 - 6 } & hcp & fcc & & hcp & fcc \\
\hline $\mathrm{a}(\AA)$ & 2.4850 & 3.5283 & & 2.5071 & 3.5442 \\
$\mathrm{~b}(\AA)$ & 2.4850 & 3.5283 & & 2.5071 & 3.5442 \\
$\mathrm{c}(\AA)$ & 4.0327 & 3.5283 & & 4.0686 & 3.5442 \\
$\alpha, \beta, \gamma$ & $90,90,120$ & $90,90,90$ & & $90,90,120$ & $90,90,90$ \\
\hline $\mathrm{x}$ & 0.3427 & 0.0030 & & 0.3333 & 0.0000 \\
$\mathrm{y}$ & 0.6588 & 0.0020 & & 0.6667 & 0.0000 \\
$\mathrm{z}$ & 0.2500 & 0.0000 & & 0.2500 & 0.0000 \\
\hline \hline
\end{tabular}

harmonics inside the spheres are expanded up to $l_{\max }=10$ while the charge density is Fourier expanded up to $G_{\max }=16$. The energy convergence has been achieved by expanding the basis function up to $R_{M T} . K_{\max }=7$, where $R_{M T}$ is the smallest atomic sphere radius in the unit cell and $K_{\max }$ gives the magnitude of the largest $\vec{k}$ vector in the plane wave expansion. The generalized gradient approximation (GGA) parametrized by Perdew-Burke-Ernzerhof (PBE) $)^{27-29}$ was used for treated the electronic exchange-correlation energy. The energy of $-7 \mathrm{Ry}$ was set to separate core and valence states. It was selected a $8 \times 8 \times 8 \mathrm{k}$ point mesh (for fcc structure) and $8 \times 8 \times 6 \mathrm{k}$ point mesh (for hcp structure) to sample the entire Brillouin-zone (BZ), yielding 29 points (for fcc structure) and 40 points (for hcp structure) in the irreducible Brillouin-zone. The structure was relaxed according to Hellmann-Feynman forces calculated at the end of each self-consistent cycle, until the forces acting on all atoms were less than $0.068 \mathrm{eV} / \AA(5 \mathrm{mRy} / \mathrm{a} . u \text {. })^{30}$. The self-consistency was achieved by demanding the convergence of the integrated charge difference between last two iterations to be smaller than $10^{-5} e$. All calculations were spin-polarized and refer to zero temperature.

The theoretically optimized lattice parameters $a, b, c, \alpha, \beta$ and $\gamma$ and fractional coordinates of atoms are presented in table 3 (second and third column). From table 3 , it can be seen that our calculated parameters are in very good agreement with the previous results (fourth and fifth column). After obtaining the optimized structural parameters, we construct $3 \times 3 \times 3$ supercell (for fcc structure) and $4 \times 4 \times 4$ supercell (for hcp structure) as shown in figure 7 . To simulate a dopant in the crystal lattice we replaced Co by a Ta atom at the non-equivalent host site of $\mathrm{Co}$, preserving the point group symmetry around the original atom. Consequently, the number of non-equivalent positions increased. The number of non-equivalent positions for fcc structures with $3 \times 3 \times 3$ supercell is now 10 and for hcp structure with $4 \times 4 \times 4$ supercell is now 25 . Due to the local group symmetry in our calculations, we do not need to consider all of these non-equivalent positions. In fact, it is enough to consider 2 non-equivalent positions for $\mathrm{fcc}$ and for hcp structures. These substitutional structural positions have been marked as Co-Ta1 and Co-Ta2 (figure 7). We have checked that the two Ta atoms are sufficiently far from each other $(\sim 10 \AA)$ to avoid significant impurity-impurity interactions. We have repeated the calculations for each substitutional structure, keeping all parameters and charge convergence criteria same as in the case of pure compounds, except that we have now selected a $3 \times 3 \times 3 \mathrm{k}$ point mesh 


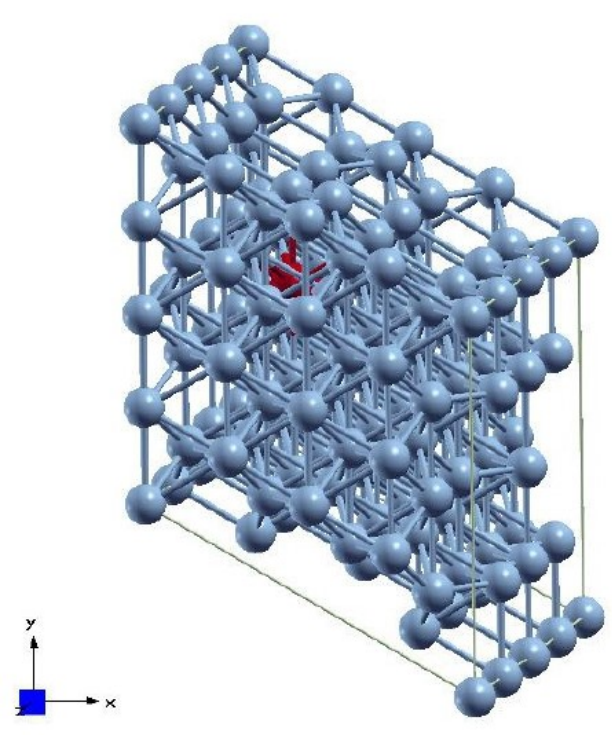

Co-Ta1 in hcp

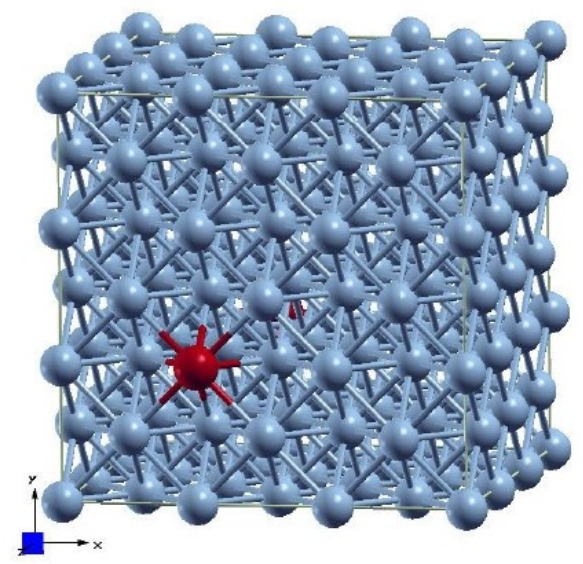

Co-Ta1 in fcc

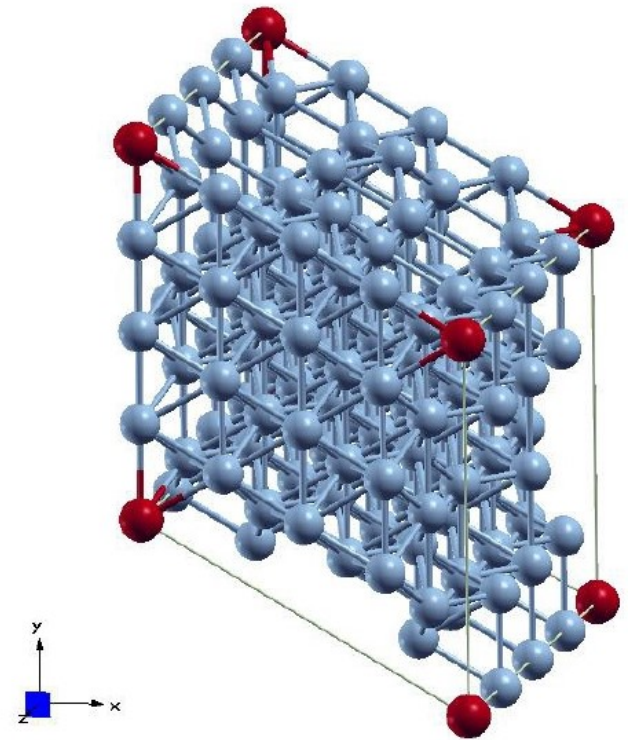

Co-Ta2 in hcp

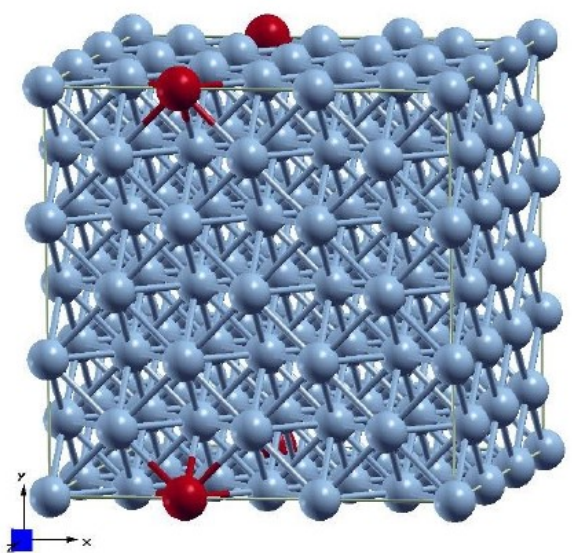

Co-Ta2 in fcc structure

Figure 7. The different supercell models with a Co atom (blue colour) substituted by Ta probe (maroon colour) used for DFT calculations. 
Table 4. The calculated $V_{z z}$ values in units of $10^{21} \mathrm{~V} / \mathrm{m}^{2}$, the asymmetry parameter $\eta$ values and the strength of the hyperfine magnetic field $B_{h f}$ for cobalt hcp $P 63 / m m c$ and fcc $F m \overline{3} m$ crystal structure.

\begin{tabular}{|c|c|c|c|c|}
\hline \multirow[b]{2}{*}{ Probe } & \multicolumn{4}{|c|}{ hcp $P 63 / m m c$} \\
\hline & Lattice Site & $V_{z z}$ & $\eta$ & $B_{h f}(\mathrm{~T})$ \\
\hline $\begin{array}{l}\text { No probe } \\
\text { (pure comp.) }\end{array}$ & Co1 & -0.18 & 0.00 & -8.73 \\
\hline \multirow[t]{4}{*}{${ }^{181} \mathrm{Ta}$} & Co-Ta1 & -6.64 & 0.71 & -25.75 \\
\hline & $\mathrm{Co}-\mathrm{Ta} 2$ & -2.25 & 0.00 & -31.97 \\
\hline & \multicolumn{4}{|c|}{ fcc $F m \overline{3} m$} \\
\hline & Lattice Site & $V_{z z}$ & $\eta$ & $B_{h f}(\mathrm{~T})$ \\
\hline $\begin{array}{l}\text { No probe } \\
\text { (pure comp.) }\end{array}$ & $\mathrm{Co} 1$ & - & - & -8.00 \\
\hline \multirow[t]{2}{*}{${ }^{181} \mathrm{Ta}$} & Co-Ta1 & 0.9543 & 0.00 & -19.99 \\
\hline & $\mathrm{Co}-\mathrm{Ta} 2$ & 0.024 & 0.17 & -21.85 \\
\hline
\end{tabular}

(for fcc structure) and $5 \times 5 \times 3 \mathrm{k}$ point mesh (for hcp structure) to sample the entire Brillouin-zone (BZ), yielding 14 points (for fcc structure) and 15 points (for hcp structure) in the irreducible Brillouin-zone.

The calculated values of $V_{z z}$, asymmetry parameter $(\eta)$ and the strength of the hyperfine magnetic field $\left(B_{h f}\right)$ in the pure compounds as well as at Ta probe positions in the fcc and hcp Co structures are given in table 4. The calculation of EFG were performed by using the method developed in Ref. ${ }^{31}$; which is implemented in WIEN2k code.

\section{Conclusion}

Both hcp and fcc crystal structures of Co have been found to be present in the temperature range 77-973 K. At room temperature, the hcp magnetic fraction is found to be dominant. The total hcp fraction is also found to be maximum at low temperature ( $298 \mathrm{~K}$ and below) as expected. But, at high temperature ( $\geq 873 \mathrm{~K}$ ), the hcp phase is again found to be predominant which is unexpected from existing knowledge and also from theoretical considerations. The fcc phase is found to be stabilized in the intermediate temperature $(\sim 500 \mathrm{~K})$ where the ferromagnetism does not change much compared to the room temperature value. It is found that at high temperature, the nonmagnetic hcp phase is more stable than the ferromagnetic fcc phase and contradicts with the results found from theoretical calculations ${ }^{8}$. It appears that magnetism does not play any role in the phase stabilization of $\mathrm{Co}$ and some other mechanism is responsible for its temperature dependent phase transformation behavior. Therefore, to explain the hcp phase stability at high temperature, and the transformation of phases in the temperature range (77-500 K) where there is almost no changes of magnetism, present experimental results demand a new theoretical interpretation which remains unexplored till now.

\section{Data Availability}

The datasets generated during and/or analysed during the current study are available from the corresponding author on reasonable request.

\section{References}

1. Nishizawa, T. \& Ishida, K. The Co (Cobalt) system. Bull. Alloy. Phase Diagrams 4, 387-390, DOI: 10.1007/BF02868089 (1983).

2. Lindgren, B., Bedi, S. \& Wäppling, R. Hyperfine Interactions of Cd Impurities in Co-metal. Phys. Scripta 18, 26-30, DOI: 10.1088/0031-8949/18/1/007 (1978).

3. Bedi, S. C. \& Forker, M. Hyperfine interactions at Ta impurities in cobalt and cobalt-hafnium intermetallic compounds. Phys. Rev. B 47, 14948-14960, DOI: 10.1103/PhysRevB.47.14948 (1993). 
4. Min, B. I., Oguchi, T. \& Freeman, A. J. Structural, electronic, and magnetic properties of Co: Evidence for magnetismstabilizing structure. Phys. Rev. B 33, 7852-7854, DOI: 10.1103/PhysRevB.33.7852 (1986).

5. Moruzzi, V. L., Marcus, P. M., Schwarz, K. \& Mohn, P. Ferromagnetic phases of bcc and fcc Fe, Co, and Ni. Phys. Rev. B 34, 1784-1791, DOI: 10.1103/PhysRevB.34.1784 (1986).

6. Zener, C. Phase stability in Metals and Alloys (McGraw-Hill, New York, 1967).

7. Uhl, M. \& Kübler, J. Exchange-Coupled Spin-Fluctuation Theory: Application to Fe, Co, and Ni. Phys. Rev. Lett. 77, 334-337, DOI: 10.1103/PhysRevLett.77.334 (1996).

8. Lizárraga, R. et al. First Principles Theory of the hcp-fcc Phase Transition in Cobalt. Sci. Reports 7, 3778, DOI: 10.1038/s41598-017-03877-5 (2017).

9. Söderlind, P., Ahuja, R., Eriksson, O., Wills, J. M. \& Johansson, B. Crystal structure and elastic-constant anomalies in the magnetic 3d transition metals. Phys. Rev. B 50, 5918-5927, DOI: 10.1103/PhysRevB.50.5918 (1994).

10. Yoo, C.-S., Söderlind, P. \& Cynn, H. The phase diagram of cobalt at high pressure and temperature: the stability of $\gamma(\mathrm{fcc})$-cobalt and new $\varepsilon^{\prime}(\mathrm{dhcp})$-cobalt. J. Physics: Condens. Matter 10, L311-L318, DOI: 10.1088/0953-8984/10/20/001 (1998).

11. Kübler, J. Theory of itinerant electron magnetism (Oxford University Press, Oxford, 2000).

12. Frauenfelder, H. \& Steffen, R. M. Alpha-,Beta- and Gamma-Ray Spectroscopy Vol. 2, edited by K. Siegbahn, (North-Holland Publishing Company, Amsterdam, 1965).

13. Schatz, G. \& Weidinger, A. Nuclear condensed matter physics Nuclear methods and applications (J Wiley and Sons, United Kingdom, 1996).

14. Perturbed angular correlation spectroscopy - A tool for the study of defects and diffusion at the atomic scale. Defect Diffusion Forum 311, 3-38, DOI: 10.4028/www.scientific.net/DDF.311.3 (2011).

15. Firestone, R. B., V. S. Shirley, C. M. B., Chu, S. Y. F. \& Zipkin, J. The $8^{\text {th }}$ edition of the Table of Isotopes (Springer, Hungary, 1999).

16. Dey, C. C. A perturbed angular correlation spectrometer for material science studies. Pramana - J. Phys. 70, 835-846, DOI: $10.1007 / \mathrm{s} 12043-008-0093-1$ (2008).

17. Boström, L., Karlsson, E. \& Zetterlund, S. Calculation of Differential Angular Correlation Factors for Non-coaxial Electric and Magnetic Fields. Phys. Scripta 2, 65-69, DOI: 10.1088/0031-8949/2/1-2/013 (1970).

18. Abragam, A. \& Pound, R. V. Influence of Electric and Magnetic Fields on Angular Correlations. Phys. Rev. 92, 943-962, DOI: 10.1103/PhysRev.92.943 (1953).

19. Matthias, E., Schneider, W. \& Steffen, R. M. Nuclear Level Splitting Caused by a Combined Electric Quadrupole and Magnetic Dipole Interaction. Phys. Rev. 125, 261-268, DOI: 10.1103/PhysRev.125.261 (1962).

20. WINFIT version 3.03, April 2005, www.uni-leipzig.de/nfp/ .

21. Motta, A. T. et al. Defects and magnetic hyperfine fields in $\mathrm{ZrFe}_{2}$ investigated using perturbed-angular-correlation spectroscopy. Phys. Rev. B 60, 1188-1196, DOI: 10.1103/PhysRevB.60.1188 (1999).

22. Sewak, R. \& Dey, C. C. Search for ferromagnetism in $\mathrm{Hf}_{2} \mathrm{Co}_{7}$ : An investigation by perturbed angular correlation (PAC) spectroscopy. J. Magn. Magn. Mater. 498, 166105, DOI: https://doi.org/10.1016/j.jmmm.2019.166105 (2020).

23. Sewak, R., Dey, C. C. \& Toprek, D. Ferromagnetism in intermetallic Hf6Co23 alloy. J. Magn. Magn. Mater. 534, 168042, DOI: https://doi.org/10.1016/j.jmmm.2021.168042 (2021).

24. Blaha, P., Schwarz, K. \& Madsen, G. K. H. (2001).

25. Available at:. http://www.crystallography.net/cod/9008492.html. Accessed: 09 May 2021.

26. Available at:. http://www.crystallography.net/cod/9010968.html. Accessed: 09 May 2021.

27. Perdew, J. P., Burke, K. \& Ernzerhof, M. Generalized gradient approximation made simple. Phys. Rev. Lett. 77, 3865-3868, DOI: 10.1103/PhysRevLett.77.3865 (1996).

28. Perdew, J. P., Burke, K. \& Ernzerhof, M. Erratum: Generalized gradient approximation made simple (Physical Review Letters (1996) 77 (3865)). Phys. Rev. Lett. 78, 1396, DOI: 10.1103/PhysRevLett.78.1396 (1997).

29. Zhang, Y. \& Yang, W. Comment on "Generalized Gradient Approximation Made Simple". Phys. Rev. Lett. 80, 890, DOI: 10.1103/PhysRevLett.80.890 (1998). 
30. Belošević-Čavor, J., Koteski, V. \& Radaković, J. Structure identification and site preference of Ta and Cd in Ti-Pd alloys: A first-principle study. Solid State Commun. 152, 1072-1075, DOI: https://doi.org/10.1016/j.ssc.2012.03.039 (2012).

31. Blaha, P., Schwarz, K. \& Herzig, P. First-Principles Calculation of the Electric Field Gradient of Li 3 N. Phys. Rev. Lett. 54, 1192-1195, DOI: 10.1103/PhysRevLett.54.1192 (1985).

\section{Acknowledgements}

The work has been carried out by the support of Department of Atomic Energy, India through the grant no. 12 R\&D-SIN-5.020102 .

\section{Author contributions statement}

R.S. and C.C.D. conceived, conducted experiment and analysed the results. D.T. performed DFT calculations. All authors contributed writing the manuscript.

\section{Competing interests}

The authors declare no competing interests.

\section{Additional information}

Correspondence and requests for materials should be addressed to C.C.D. 